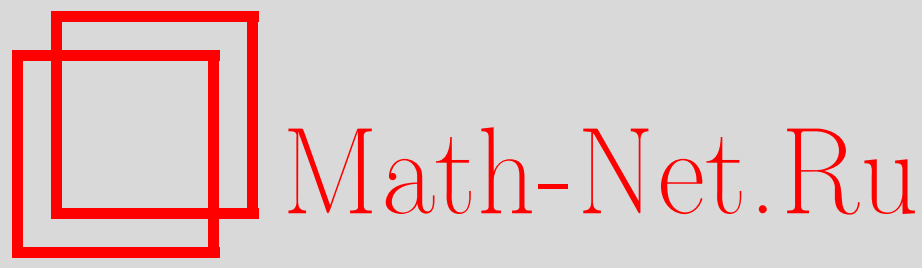

В. В. Пупышев, Кулоновское рассеяние медленной квантовой частицы в пространстве произвольной размерности, ТМФ, 2018, том 195, номер 1, 64-74

DOI: https://doi.org/10.4213/tmf9308

Использование Общероссийского математического портала Math-Net.Ru подразумевает, что вы прочитали и согласны с пользовательским соглашением http://www.mathnet.ru/rus/agreement

Параметры загрузки:

IP : 34.239 .49 .27

26 апреля 2023 г., 15:03:41

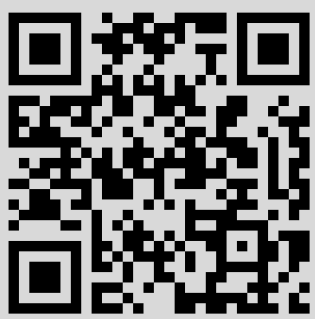




\title{
КУЛОНОВСКОЕ РАССЕЯНИЕ МЕДЛЕННОЙ КВАНТОВОЙ ЧАСТИЦЫ В ПРОСТРАНСТВЕ ПРОИЗВОЛЬНОЙ РАЗМЕРНОСТИ
}

\begin{abstract}
Считается, что заряженная квантовая частица движется в пространстве размерности $d=2,3, \ldots$ и рассеивается неподвижным кулоновским центром. Выведены и исследованы разложения волновой функции и всех радиальных волновых функций такой частицы по целым степеням волнового числа и функциям Бесселя вещественного порядка. Доказано, что конечные суммы таких разложений являются асимптотиками волновых функций в пределе низких энергий.
\end{abstract}

Ключевые слова: кулоновское рассеяние, волновые функции, низкоэнергетические асимптотики.

DOI: https://doi.org/10.4213/tmf9308

\section{1. ВВЕДЕНИЕ}

Явные представления волновой функции $\Psi_{\mu}^{\mathrm{c}}$ и радиальной волновой функции $F_{\lambda}$ кулоновского рассеяния квантовой частицы $p_{1}$ в координатном пространстве $\mathbb{R}^{d}$ произвольной размерности $d=2,3, \ldots$ впервые получены в работе [1]. Низкоэнергетические асимптотики функций $\Psi_{\mu}^{\mathrm{c}}$ и $F_{\lambda}$ известны [2] только в случае $d=2$.

Главная цель настоящей работы - вывод и анализ таких асимптотик при любом $d \geqslant 2$. В разделе 2 поясняются обозначения и основные определения. В разделе 3 известные представления [1] функций $\Psi_{\mu}^{c}$ и $F_{\lambda}$ через функции Куммера [3] записываются в обозначениях, принятых в разделе 2 . В разделе 4 выводятся и исследуются низкоэнергетические аппроксимации волновых функций $\Psi_{\mu}^{c}$ и $F_{\lambda}$. В заключении перечисляются основные результаты.

Настоящая работа является сокращенной версией препринта [4].

\section{2. ОПРЕДЕЛЕНИЯ И ОБОЗНАЧЕНИЯ}

Поясним используемые в настоящей работе обозначения и определения, а затем приведем соотношения, известные в теории высших трансцендентных функций [3] и в теории гипергармоник [5], [6].

* Объединенный институт ядерных исследований, Дубна, Московская обл., Россия. E-mail: pupyshev@theor.jinr.ru 
Предположим, что квантовая частица $p_{1}$ имеет массу $m_{1}$, обладает кулоновским зарядом $z_{1}$ и движется в координатном пространстве $\mathbb{R}^{d}$ некоторой размерности $d \geqslant 2$. В этом пространстве введем правую декартову систему координат $S^{d}$ с начальной точкой $O$ и единичными направляющими ортами $\mathbf{e}_{1}, \mathbf{e}_{2}, \ldots, \mathbf{e}_{d}$, один из которых коллинеарен волновому вектору $\mathbf{k}_{0}$ начального состояния частицы $p_{1}$. Пусть $\mathbf{r}$ - ее радиус-вектор. Используем его гиперсферические координаты $(r, \Omega)$, где $r$ гиперрадиус, а $\Omega$ - некоторый набор всех гиперсферических углов.

Считаем, что точка $O$ является неподвижным силовым центром, который имеет кулоновский заряд $Z$ и воздействует на частицу $p_{1}$ посредством кулоновского потенциала $V^{\mathrm{c}}(r)=z_{1} Z / r$. Символом $\mathbf{k}$ обозначим волновой вектор конечного состояния частицы $p_{1}$, а углом ее рассеяния назовем угол $\theta$ между векторами $\mathbf{k}_{0}$ и $\mathbf{r}$. Сохраним все принятые в работе [2] обозначения:

$$
\begin{array}{rlrlrl}
k & =\sqrt{\frac{2 m_{1} E}{\hbar^{2}}}, & R \equiv \frac{\hbar^{2}}{2 m_{1} z_{1} Z}, & q & =k|R|, & \alpha \equiv \operatorname{sgn} R, \\
\eta \equiv \frac{1}{2 k R}=\frac{\alpha}{2 q}, & \rho \equiv k r, & v \equiv \sqrt{8|\eta| \rho}, & w \equiv v \sin \frac{\theta}{2} .
\end{array}
$$

Здесь и далее $\hbar$ - постоянная Планка, $E$ - полная энергия частицы $p_{1}$, а $k$ и $q$ - ее размерное и безразмерное волновые числа, $\eta$ - параметр Зоммерфельда, $\rho, v$ и $w-$ безразмерные неотрицательные переменные. Согласно принятым обозначениям

$$
\rho=q \frac{v^{2}}{4}=\frac{q \omega^{2}}{2(1-\cos \theta)} .
$$

Для краткости используем символы и формулы с верхними и нижними знаками плюс и минус. В таких формулах все верхние знаки берутся в случае кулоновского отталкивания $(\alpha=1)$, а все нижние - в случае кулоновского притяжения $(\alpha=-1)$. Символами $Z_{\mathrm{c}}^{+}(t)$ и $Z_{\mathrm{c}}^{-}(t)$ обозначим функции Бесселя $I_{\mathrm{c}}(t)$ и $J_{\mathrm{c}}(t)$. Если $W$ - некоторый бесконечный ряд с общим множителем $g$ и слагаемыми $w_{n}$, то символом $W_{m}$ обозначаем конечную $(n=0,1, \ldots, m, m<\infty)$ подсумму этого ряда:

$$
W=g \sum_{n=0}^{\infty} w_{n}, \quad W_{m} \equiv g \sum_{n=0}^{m} w_{n} .
$$

Согласно теории гипергармоник [5], [6] в пространстве $\mathbb{R}^{d}$ гипергармоники $Y_{L M}(\Omega)$ являются собственными функциями квадрата $\mathbf{L}^{2}(\Omega)$ оператора гипермомента $\mathbf{L}(\Omega)$ :

$$
\mathbf{L}^{2} Y_{L \mathbf{M}}(\Omega)=L(L+d-2) Y_{L \mathbf{M}}(\Omega), \quad L=0,1, \ldots
$$

Гипергармоники образуют полный и ортонормированный базис в пространстве функций, квадратично суммируемых на единичной сфере $\mathbb{S}^{d-1}$ с центром в точке $O$. Оператор $-\mathbf{L}^{2}(\Omega)$ равен угловой части оператора Лапласа, которая часто называется оператором Лапласа-Бельтрами в пространстве $\mathbb{R}^{d}$. Если $d \geqslant 3$, то множество М содержит все квантовые числа гипергармоники $Y_{L M}$, за исключением гипермомента $L$. Количество таких чисел равно $d-2$. Поэтому в случае $d=2$ множество $\mathbf{M}$ пусто. 
Напомним, что функция Куммера $\Phi(a, c ; \xi)$ удовлетворяет тождеству (преобразованию) Куммера [3]

$$
\Phi(a, c ; \xi) \equiv e^{\xi} \Phi(c-a, c ;-\xi) .
$$

Введем функции $\mu, \lambda$ и $\nu$ дискретных переменных $d$ и $L$. По определению

$$
\mu \equiv \frac{d-3}{2}, \quad d=2,3, \ldots ; \quad \lambda \equiv L+\mu, \quad \nu \equiv 2 \lambda+1, \quad L=0,1, \ldots
$$

Поэтому $\mu$ и $\lambda$ - полуцелые числа при четном $d$ и целые при нечетном $d$, причем $\mu>0$ и $\lambda>0$, если $d \geqslant 4$. Два случая $d=2$ и $d=3$ являются особыми. Если $d=2$, то $\mu=-1 / 2$, а $\lambda=-1 / 2$ при $L=0$. Если $d=3$, то $\mu=0$, а $\lambda=0$ только при $L=0$.

Под низкоэнергетической асимптотикой исследуемой функции подразумеваем ее асимптотику в пределе нулевой энергии рассеяния $(E \rightarrow 0+)$ при фиксированных значениях отличного от нуля кулоновского параметра $R$ и порядков $\mu$ и $\lambda$. В таком пределе $q \rightarrow 0+$, a $|\eta| \rightarrow \infty$.

\section{3. ИСХОДНЫЕ ПРЕДСТАВЛЕНИЯ ВОЛНОВЫХ ФУНКЦИЙ}

Исходными для наших исследований являются известные представления [1] полной и радиальной волновых функций $\Psi_{\mu}^{c}$ и $F_{\lambda}$ упругого кулоновского рассеяния частицы $p_{1}$ в пространстве $\mathbb{R}^{d}$ произвольной размерности $d \geqslant 2$. Запишем такие представления в обозначениях, принятых в предыдущем разделе.

Волновая функция $\Psi_{\mu}^{\mathrm{c}}$ кулоновского рассеяния частицы $p_{1}$ в пространстве $\mathbb{R}^{d}$ определяется как непрерывное в области $\{\rho \geqslant 0,0<\theta \leqslant \pi\}$ решение $d$-мерного уравнения Шредингера

$$
\left[\partial_{\rho}^{2}+\frac{d-1}{\rho} \partial_{\rho}-\frac{\mathbf{L}^{2}(\Omega)}{\rho^{2}}+1-\frac{2 \eta}{\rho}\right] \Psi_{\mu}^{\mathrm{c}}(\rho, \theta, \eta)=0, \quad \eta \in(-\infty, \infty),
$$

с граничным условием

$$
\begin{aligned}
& \Psi^{\mathrm{c}}(\rho, \theta, \eta)=e^{i \rho \cos \theta+i \eta \ln [\rho(1-\cos \theta)]}+ \\
& \quad+r^{-\mu-1} e^{i \rho-i \eta \ln (2 \rho)} f^{\mathrm{c}}(\theta, \eta)+O\left(\rho^{-\mu-2}\right), \quad \rho \rightarrow \infty, \quad \theta \neq 0 .
\end{aligned}
$$

Такое решение содержит искомую амплитуду кулоновского рассеяния $f^{c}$ и записывается в виде произведения

$$
\Psi_{\mu}^{\mathrm{c}}(\rho, \theta, \eta)=B_{\mu}(\eta) e^{i \rho} \Phi(\mu+1+i \eta, \mu+1 ; \xi), \quad \xi=i \rho(\cos \theta-1),
$$

в котором

$$
B_{\mu}(\eta) \equiv \frac{\Gamma(\mu+1+i \eta)}{\Gamma(\mu+1)} e^{-\pi \eta / 2} .
$$

Представление (4) после преобразования Куммера (2) принимает вид

$$
\Psi_{\mu}^{\mathrm{c}}(\rho, \theta, \eta)=B_{\mu}(\eta) e^{i \rho \cos \theta} \Phi(-i \eta, \mu+1 ;-\xi) .
$$

В уравнении (3) кулоновский потенциал $2 \eta / \rho$ не зависит ни от одного гиперугла из полного набора $\Omega$. Поэтому это уравнение подстановкой

$$
\Psi_{\mu}^{\mathrm{c}}(\rho, \theta, \eta)=\rho^{-\mu-1} \sum_{\lambda=\mu}^{\infty} i^{\lambda} e^{i \delta_{\lambda}^{\mathrm{c}}(\eta)} F_{\lambda}(\rho, \eta) \sum_{\mathbf{M}} Y_{L \mathbf{M}}(\Omega)
$$


сводится к счетной $(\lambda=L+\mu, L=0,1, \ldots)$ совокупности уравнений Кулона

$$
\left[\partial_{\rho}^{2}-\frac{\lambda(\lambda+1)}{\rho^{2}}+1-\frac{2 \eta}{\rho}\right] F_{\lambda}(\rho, \eta)=0, \quad \rho>0, \quad \eta \in(-\infty, \infty),
$$

с условиями $F_{\lambda} \sim \rho^{\lambda+1}, \rho \rightarrow 0$, и условиями

$$
F_{\lambda}(\rho, \eta)=\sin \left[\rho-\eta \ln (2 \rho)-\frac{\pi \lambda}{2}+\delta_{\lambda}^{\mathrm{c}}(\eta)\right]+O\left(\frac{1}{\rho}\right), \quad \rho \rightarrow \infty,
$$

в которых символом $\delta_{\lambda}^{\mathrm{c}}(\eta)$ обозначен аргумент гамма-функций $\Gamma(\lambda+1+i \eta)$.

Решение $F_{\lambda}$ уравнения Кулона с такими условиями является произведением

$$
F_{\lambda}(\rho, \eta)=C_{\lambda}(\eta) \rho^{\lambda+1} e^{i \rho} \Phi(\lambda+1+i \eta, 2 \lambda+2 ;-2 i \rho),
$$

в котором

$$
C_{\lambda}(\eta) \equiv 2^{\lambda} \frac{|\Gamma(\lambda+1+i \eta)|}{\Gamma(2 \lambda+2)} e^{-\pi \eta / 2} .
$$

Это произведение считается регулярной радиальной волновой функцией кулоновского рассеяния частицы $p_{1}$ в состоянии $|q, \lambda, \mathbf{M}\rangle$ с квантовыми числами $q, \lambda$ и $\mathbf{M}$.

\section{4. НИЗКОЭНЕРГЕТИЧЕСКИЕ АСИМПТОТИКИ}

Наша главная задача - построить низкоэнергетические асимптотики функции $\Psi_{\mu}^{c}$ и $F_{\lambda}$ в виде конечных сумм $\Psi_{\mu m}^{\mathrm{c}}$ и $F_{\lambda m}$ бесконечных рядов, содержащих вполне определенные коэффициенты $a_{n s}, b_{n s}, c_{n s}, d_{n s}, p_{n s}, t_{n s}$, функции Бесселя $Z_{b}^{ \pm}(t)$ вещественного порядка $b$, целые степени $t^{s}$ аргумента $t=\omega$ или $t=v$ и целые степени $q^{n}$ волнового числа $q$.

Представления (4)-(6) и (8), (9) функций $\Psi_{\mu}^{\mathrm{c}}$ и $F_{\lambda}$ содержат в качестве множителей экспоненциальные функций и функции Куммера. Поэтому функции $\Psi_{\mu}^{c}$ и $F_{\lambda}$ можно представить в виде бесконечных рядов двух типов. Разложения первого типа содержат экспоненцильные множители, в разложениях второго типа такие множители отсутствуют. Разложения обоих типов выводятся по однотипной схеме. Сначала в уравнении Шредингера (3) или в уравнении Кулона (7) волновая функция $\Psi_{\mu}^{c}$ или $F_{\lambda}$ заменяется искомым бесконечным рядом, содержащим известные функции и заранее неизвестные коэффициенты $a_{n s}, b_{n s}, c_{n s}, d_{n s}, p_{n s}, t_{n s}$. Затем с помощью рекуррентных соотношений для функций Бесселя [3] выводятся алгебраические уравнения, определяющие такие коэффициенты. Подробный вывод и исчерпывающий анализ разложений функций $\Psi_{\mu}^{\mathrm{c}}$ и $F_{\lambda}$ даны в препринте [4]. Поэтому ниже мы перечисляем такие разложения, а для доказательства их равномерной сходимости воспользуемся результатами работы [2]. Такую сходимость проиллюстрируем вычислениями в физически наиболее интересном случае трехмерного $(d=3, \mu=0)$ кулоновского рассеяния.

4.1. Асимптотики волновой функции. Представлению (4) функции $\Psi_{\mu}^{c}$ отвечает следующее разложение первого типа:

$$
\Psi_{\mu}^{\mathrm{c}}(\rho, \theta, \pm|\eta|)=B_{\mu}(\eta) \Gamma(\mu+1) e^{i \rho}\left(\frac{2}{w}\right)^{\mu} \sum_{n=0}^{\infty}\left(\mp i \frac{q}{2}\right)^{n} \sum_{s=n}^{2 n} a_{n s}( \pm w)^{s} Z_{\mu+s}^{ \pm}(w) .
$$


В этом разложении все коэффициенты $a_{n s}$ определяются бесконечной цепочкой рекуррентных уравнений $s a_{n s}=2(\mu+s) a_{n-1, s-1}+a_{n-1, s-2}, n=1,2, \ldots$ В этих уравнениях при каждом значении индекса $n$ полагается, что $s=n, n+1, \ldots, 2 n$, и подразумевается, что $a_{00}=1$ и $a_{n m}=0$, если $m<n$ или $m>2 n$.

Представлению (6) волновой функции $\Psi_{\mu}^{c}$ соответствует разложение первого типа

$$
\begin{aligned}
\Psi_{\mu}^{\mathrm{c}}(\rho, \theta, \pm|\eta|) & =B_{\mu}(\eta) \Gamma(\mu+1) e^{i \rho \cos \theta}\left(\frac{2}{w}\right)^{\mu}\left[Z_{\mu}^{ \pm}(w)+T_{\mu}^{ \pm}(w, q)\right] \\
T_{\mu}^{ \pm}(w, q) & \equiv \sum_{n=1}^{\infty}\left( \pm i \frac{q}{2}\right)^{n} \sum_{s=n+1}^{2 n} p_{n s}( \pm w)^{s} Z_{\mu+s}^{ \pm}(w) .
\end{aligned}
$$

Коэффициент $p_{00}$ этого разложения равен единице, а все остальные коэффициенты $p_{n s}$ удовлетворяют рекуррентной цепочке $(n=1,2, \ldots)$ уравнений

$$
\begin{aligned}
(n+1) p_{n, n+1} & =2 n p_{n-1, n}, & & s=n+1, \quad n>1, \\
s p_{n s} & =2(s-1) p_{n-1, s-1}+p_{n-1, s-2}, & & s=n+2, n+3, \ldots, 2 n-1, \\
2 n p_{n, 2 n} & =p_{n-1,2(n-1)}, & & s=2 n .
\end{aligned}
$$

Эти уравнения не содержат никаких параметров. Поэтому коэффициенты $p_{n s}$ являются универсальными в следующем смысле: при любой размерности $d$ пространства $\mathbb{R}^{d}$ разложение $(11)$ волновой функции $\Psi_{\mu}^{\mathrm{c}}$ содержит одни и те же коэффициенты $p_{n s}$. В работе [2] была доказана равномерная сходимость ряда $T^{ \pm} \equiv T_{\mu}^{ \pm}$ в области $\rho<\infty$. Следовательно, при любых $\mu=(d-3) / 2$ и $\rho<\infty$ разложение (11) сходится равномерно. Это означает, что в области $\rho<\infty$ любая, но конечная $(n=0,1, \ldots, m<\infty)$ подсумма

$$
\begin{aligned}
\Psi_{\mu m}^{\mathrm{c}}(\rho, \theta, \pm|\eta|) \equiv B_{\mu} & (\eta) \Gamma(\mu+1) e^{i \rho \cos \theta}\left(\frac{2}{w}\right)^{\mu} \times \\
\times & {\left[Z_{\mu}^{ \pm}(w)+\sum_{n=1}^{m}\left( \pm i \frac{q}{2}\right)^{n} \sum_{s=n+1}^{2 n} p_{n s}( \pm w)^{s} Z_{\mu+s}^{ \pm}(w)\right] }
\end{aligned}
$$

этого разложения является низкоэнергетической асимптотикой функции $\Psi_{\mu}^{c}$ :

$$
\Psi_{\mu}^{\mathrm{c}}(\rho, \theta, \pm|\eta|)=\Psi_{\mu m}^{\mathrm{c}}(\rho, \theta, \pm|\eta|)+O\left(B_{\mu}(\eta) e^{i \rho \cos \theta} q^{m+1}\right), \quad q \rightarrow 0, \quad \rho<\infty .
$$

Для волновой функции $\Psi_{\mu}^{\mathrm{c}}$ верно следующее разложение второго типа:

$$
\Psi_{\mu}^{\mathrm{c}}(\rho, \theta, \pm|\eta|)=B_{\mu}(\eta) \Gamma(\mu+1)\left(\frac{2}{w}\right)^{\mu} \sum_{n=0}^{\infty}\left[ \pm \frac{i q}{2(1-\vartheta)}\right]^{n} \sum_{s=n}^{2 n} t_{n s}(\vartheta)( \pm w)^{s} Z_{\mu+s}^{ \pm}(w)
$$

В этом разложении $\vartheta \equiv \cos \theta$, все функции $t_{n s}(\vartheta)$ непрерывны на отрезке $-1 \leqslant \vartheta \leqslant 1$ и определяются рекуррентной цепочкой $(n=1,2, \ldots ; s=n, n+1, \ldots, 2 n)$ уравнений

$$
\begin{gathered}
s t_{n s}(\vartheta)=2[s-1+(s+\mu) \vartheta] t_{n-1, s-1}(\vartheta)+(1+\vartheta) t_{n-1, s-2}(\vartheta)- \\
-2 \vartheta\left[2(\mu+s-1) t_{n-2, s-2}(\vartheta)+t_{n-2, s-3}(\vartheta)\right],
\end{gathered}
$$

в которой $t_{00}(\vartheta) \equiv 1$ и полагается $t_{n m}(\vartheta) \equiv 0$, если $m<n$ или $m>2 n$. 
Как упоминалось выше, разложение (11) волновой функции $\Psi_{\mu}^{c}$ сходится равномерно при любых $\mu$ и $\rho<\infty$. Следовательно, оба разложения (10) и (12) этой же функции обладают таким же свойством. Поэтому любые конечные суммы $\Psi_{\mu m}^{c}$ разложений (10) и (12) можно использовать как низкоэнергетические $(q \rightarrow 0)$ асимптотики волновой функции $\Psi_{\mu}^{\mathrm{c}}$ в области $\rho<\infty$.

4.2. Асимптотики радиальных волновых функций. При любом $\lambda=L+\mu$, $L=0,1, \ldots$, для радиальной волновой функции $F_{\lambda}$ имеет место следующее разложение первого типа:

$$
F_{\lambda}(\rho, \pm|\eta|)=q^{\lambda+1} C_{\lambda}(\eta) \Gamma(2 \lambda+2) e^{i \rho} \frac{v}{2} \sum_{n=0}^{\infty}\left(\mp i \frac{q}{2}\right)^{n} \sum_{s=n}^{2 n} c_{n s}( \pm v)^{s} Z_{2 \lambda+1+s}^{ \pm}(v)
$$

Все коэффициенты $c_{n s}$ этого разложения подчиняются рекуррентной цепочке уравнений

$$
s c_{n s}=2(\lambda+s) c_{n-1, s-1}+c_{n-1, s-2}, \quad n=1,2, \ldots
$$

В этих уравнениях при каждом значении индекса $n$ полагается $s=n, n+1, \ldots, 2 n$ и подразумевается, что $c_{00}=1$ и $c_{n m}=0$, если $m<n$ или $m>2 n$.

Для каждой функции $F_{\lambda}(\lambda=L+\mu, L=0,1, \ldots)$ верно разложение второго типа

$$
F_{\lambda}(\rho, \pm|\eta|)=q^{\lambda+1} C_{\lambda}(\eta) \Gamma(2 \lambda+2) \frac{v}{2} \sum_{n=0}^{\infty}\left(-i \frac{q}{2}\right)^{2 n} \sum_{s=2 n}^{3 n} d_{n s}( \pm v)^{s} Z_{2 \lambda+1+s}^{ \pm}(v)
$$

Коэффициенты $d_{n s}$ этого разложения удовлетворяют рекуррентным уравнениям

$$
2 s d_{n s}=2(2 \lambda+s) d_{n-1, s-2}+d_{n-1, s-3},
$$

в которых $s=2 n, 2 n+1, \ldots, 3 n$ при каждом $n=1,2, \ldots$ и по определению $d_{00}=1$, a $d_{n m}=0$, если выполнено условие $m<2 n$ или условие $m>3 n$.

Обсудим представление (14). Для этого подстановкой

$$
\nu=2 \lambda+1, \quad d_{n s}=\frac{b_{n s}}{2^{n} n !}, \quad n=1,2, \ldots, \quad s=n, n+1, \ldots, 2 n,
$$

сведем его к известному (см. [2]), но полученному другим способом разложению

$$
F_{\lambda}(\rho, \pm|\eta|)=q^{\lambda+1} C_{\lambda}(\eta) \Gamma(\nu+1) \frac{v}{2} \sum_{n=0}^{\infty}\left(-\frac{q^{2}}{8}\right)^{n} \frac{1}{n !} \sum_{s=2 n}^{3 n} b_{n s}( \pm v)^{s} Z_{\nu+s}^{ \pm}(v)
$$

Как доказано в работе [2], при условии $\rho<\infty$ такое разложение сходится абсолютно и равномерно. Следовательно, исходное разложение (14), а значит и разложение (13), обладает таким же свойством. Поэтому при условии $\rho<\infty$ любые, но конечные суммы $F_{\lambda m}$ разложений (13) и (14) являются искомыми низкоэнергетическими асимптотиками функции $F_{\lambda}$. 


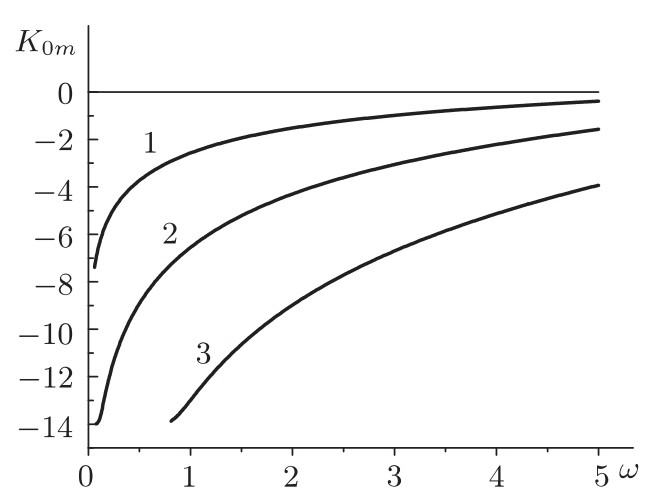

a

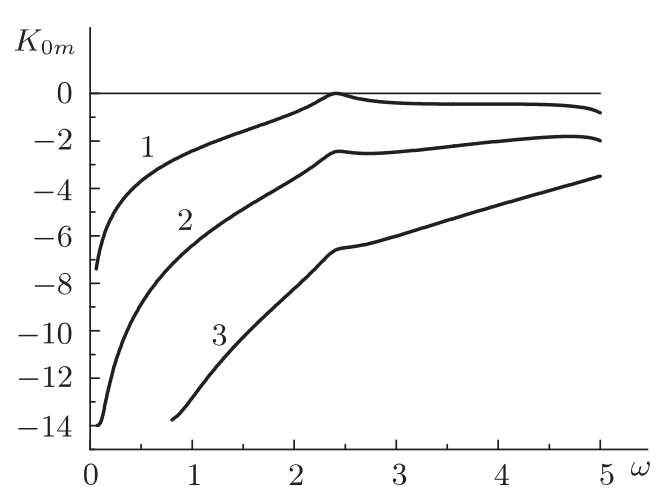

б

Рис. 1. Графики функций $K_{0 m}(\omega, \eta, \varepsilon)$ для разложения $(11) ; \varepsilon=10^{-14}$, значениям $m=0,2,5$ соответствуют кривые 1,2 и 3 при $\eta=5$ (а), при $\eta=-5$ (б).

4.3. Численный анализ приближений волновых функций. Как показано в п. 4.1 и п. 4.2 , волновые функции $\Psi_{\mu}^{\text {с }}$ и $F_{\lambda}$ являются бесконечными рядами $(10)-(12)$ и $(13),(14)$. Предложим и реализуем метод, позволяющий оценить относительную точность приближения таких рядов их конечными суммами $\Psi_{\mu m}^{\mathrm{c}}$ и $F_{\lambda m}$. В этом методе функции $\Psi_{\mu}^{c}$ и $F_{\lambda}$, вычисленные по формулам $(4),(5)$ и $(8),(9)$, сравниваются с подсуммами $\Psi_{\mu m}^{\mathrm{c}}$ и $F_{\lambda m}$ соответствующих рядов $(10)-(14)$. Для такого сравнения используются функции $K_{\mu m}, L_{\mu m}$ или $D_{\lambda m}$, близкие к десятичному логарифму относительной точности приближения $\Psi_{\mu}^{\mathrm{c}} \approx \Psi_{\mu m}^{\mathrm{c}}$ или приближения $F_{\lambda} \approx F_{\lambda m}$.

Для анализа сходимости рядов (10) и (11) предлагается использовать функцию

$$
K_{\mu m}(w, \eta, \varepsilon) \equiv \ln \frac{\left|\Psi_{\mu}^{\mathrm{c}}(\rho, \theta, \eta)-\Psi_{\mu m}^{\mathrm{c}}(\rho, \theta, \eta)\right|+\varepsilon}{\left|\Psi_{\mu}^{\mathrm{c}}(\rho, \theta, \eta)\right|+\varepsilon}, \quad \rho=\frac{q w^{2}}{2(1-\cos \theta)}, \quad \varepsilon>0 .
$$

Эта функция в пределе $\varepsilon \rightarrow 0+$ становится десятичным логарифмом относительной точности приближения $\Psi_{\mu}^{\mathrm{c}} \approx \Psi_{\mu m}^{\mathrm{c}}$, а при достаточно малом $\varepsilon$ близка к такой точности.

Рис. 1 иллюстрирует сходимость ряда (11) в случаях трехмерных $(d=3, \mu=0)$ отталкивающего $(q=0.1, \eta=5)$ и притягивающего $(q=0.1, \eta=-5)$ кулоновского рассеяний квантовой частицы $p_{1}$. На рис. 1 изображены графики трех $(m=0,2,5)$ функций $K_{\mu m}(w, \eta, \varepsilon), \mu=0, \varepsilon=10^{-14}$. Как видно, на отрезке $0 \leqslant w \leqslant 5$ относительная точность приближения $\Psi_{\mu}^{\mathrm{c}} \approx \Psi_{\mu m}^{\mathrm{c}}$ быстро улучшается с ростом числа $m$.

Результаты расчета функции $K_{\mu m}, \mu=0$, подтвердили быструю сходимость разложения (10) на отрезке $0 \leqslant w \leqslant 5$ в обоих случаях $\eta=5$ и $\eta=-5$.

Для исследования сходимости ряда (12) удобно использовать функцию

$$
L_{\mu m}(\rho, \theta, \eta, \varepsilon) \equiv \ln \frac{\left|\Psi_{\mu}^{\mathrm{c}}(\rho, \theta, \eta)-\Psi_{\mu m}^{\mathrm{c}}(\rho, \theta, \eta)\right|+\varepsilon}{\left|\Psi_{\mu}^{\mathrm{c}}(\rho, \theta, \eta)\right|+\varepsilon}, \quad \varepsilon>0 .
$$

Для примера эта функция вычислялась в случае $\theta=\pi / 4$ и при тех же значениях $\mu, m, \eta$ и $\varepsilon$, что и функция $K_{\mu m}$. Результаты вычислений представлены на рис. 2 


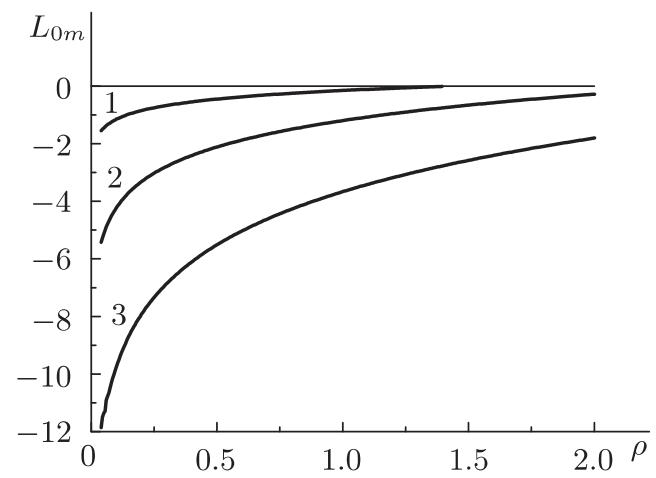

a

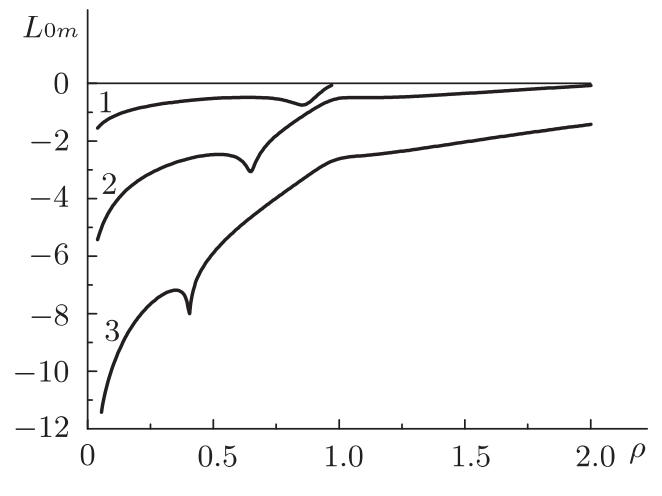

б

Рис. 2. Графики функций $L_{0 m}(\rho, \theta, \eta, \varepsilon) ; \varepsilon=10^{-14}, \theta=\pi / 4$, значениям $m=0,2,5$ соответствуют кривые 1,2 и 3 при $\eta=5$ (а), $\eta=-5$ (б).

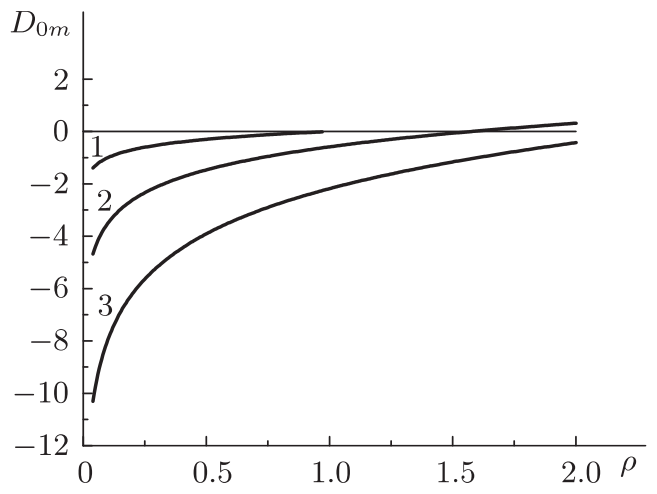

a

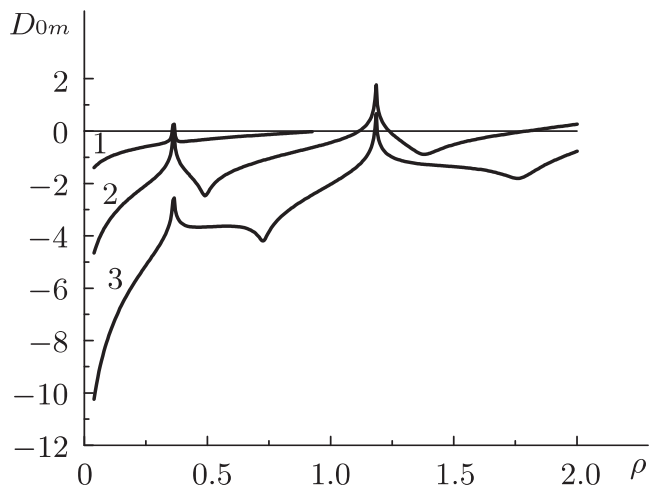

б

Рис. 3. Графики функций $D_{0 m}(\rho, \eta, \varepsilon)$ для разложения $(13) ; \varepsilon=10^{-14}$, значениям $m=0,2,5$ соответствуют кривые 1,2 и 3 при $\eta=5$ (a), $\eta=-5$ (б).

в виде графиков, иллюстрирующих сходимость частичных сумм $\Psi_{\mu m}^{c}$ ряда $(12)$ при $\mu=0$ к этому ряду с ростом числа $m$ при условии $\rho \leqslant 5$.

Для исследования сходимости обоих разложений (13) и (14) радиальной волновой функции $F_{\lambda}$ выгодно вычислять функцию

$$
D_{\lambda m}(\rho, \eta, \varepsilon) \equiv \ln \frac{\left|F_{\lambda}(\rho, \eta)-F_{\lambda m}(\rho, \eta)\right|+\varepsilon}{\left|F_{\lambda}(\rho, \eta)\right|+\varepsilon}, \quad \varepsilon>0
$$

Функция $D_{\lambda m}$ равна нулю в точке $\rho=0$ и имеет локальный максимум или локальный минимум в той точке, в которой функция $F_{\lambda}$ или разность функций $F_{\lambda}$ и $F_{\lambda m}$ обращается в нуль. При достаточно малом, но положительном значении аргумента $\varepsilon$ обсуждаемая функция $D_{\lambda m}$ близка к десятичному логарифму относительной точности аппроксимации $F_{\lambda} \approx F_{\lambda m}$. 

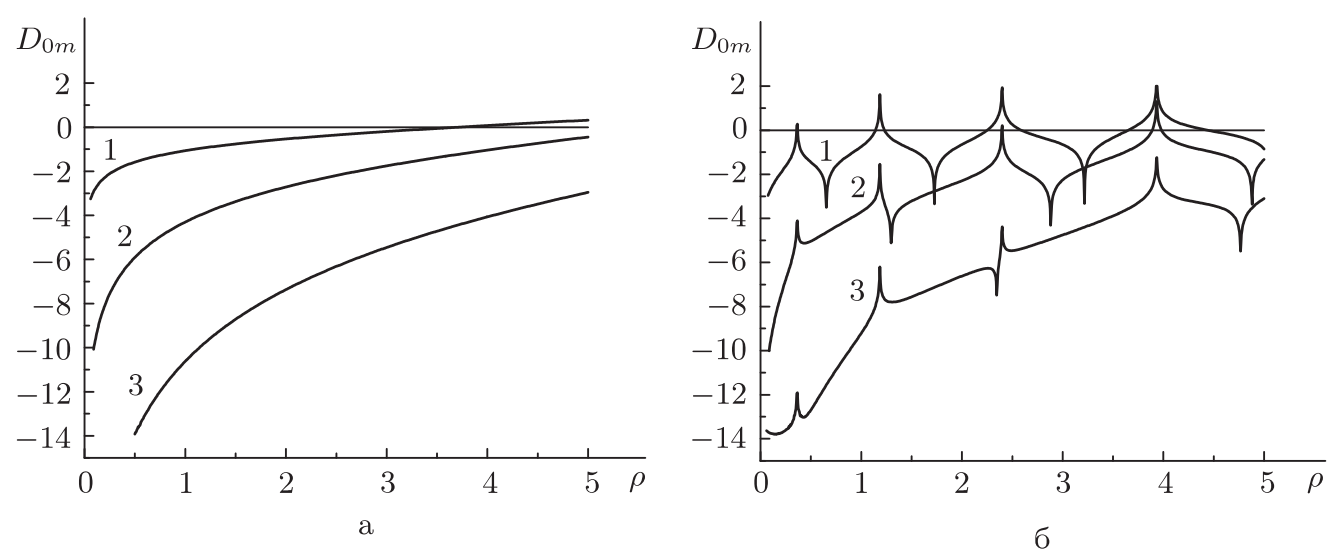

Рис. 4. Графики функций $D_{0 m}(\rho, \eta, \varepsilon)$ для разложения $(14) ; \varepsilon=10^{-14}$, значениям $m=0,2,5$ соответствуют кривые 1,2 и 3 при $\eta=5$ (а), при $\eta=-5$ (б).

Рис. 3 дает наглядное представление об относительной точности приближения функции $F_{\lambda}$ конечной суммой $F_{\lambda m}$ разложения (13). На этом рисунке изображены графики трех $(m=0,2,5)$ функций $D_{\lambda m}(\rho, \eta, \varepsilon), \varepsilon=10^{-14}, \eta= \pm 5$, вычисленных в случае трехмерного кулоновского рассеяния квантовой частицы $p_{1}$ в состоянии $|q, \lambda\rangle$ с нулевым угловым моментом $\lambda=0$.

Для иллюстрации сходимости ряда (14) функции $D_{\lambda m}(\rho, \eta, \varepsilon), m=0,2,5$, вычислялись в случае $\varepsilon=10^{-14}, \eta= \pm 5$ и $\lambda=0$. Графики таких функций представлены на рис. 4.

4.4. Асимптотики кулоновских множителей и парциальных фаз. Выведем и обсудим низкоэнергетические $(q \rightarrow 0,|\eta| \rightarrow \infty)$ асимптотики кулоновской фазы $\delta_{\lambda}^{\text {c }}$ и множителей $B_{\mu}$ и $C_{\lambda}$, заданных формулами $(5)$ и (9). В качестве ключевых равенств используем известные свойства гамма-функции [3]

$$
\Gamma(t+1)=t \Gamma(t), \quad|\Gamma(1 / 2+i \eta)|=\sqrt{\frac{\pi}{\operatorname{ch} \pi \eta}}, \quad|\Gamma(i \eta)|=\sqrt{\frac{\pi \eta}{\operatorname{sh} \pi \eta}},
$$

формулу Муавра $t=|t| e^{i \arg t}$ и формулу Стирлинга

$$
\Gamma(t)=\sqrt{2 \pi} \exp [-t+(t-1 / 2) \ln t]\left(1+O\left(t^{-1}\right), \quad|t| \rightarrow \infty, \quad|\arg t|<\pi .\right.
$$

По определению фаза $\delta_{\lambda}^{c}$ равна аргументу гамма-функции $\Gamma(t)$ переменной

$$
t \equiv \lambda+1+i \eta=\sqrt{(\lambda+1)^{2}+\eta^{2}} \exp \left[i \operatorname{arctg} \frac{\eta}{\lambda+1}\right] .
$$

В рассматриваемом пределе модуль переменой $t$ неограниченно возрастает. Поэтому можно применить формулу Стирлинга и затем получить искомую асимптотику

$$
\delta_{\lambda}^{\mathrm{c}}(\eta)=\left\{\frac{\eta}{2} \ln \left[(\lambda+1)^{2}+\eta^{2}\right]-\eta+\left(\lambda+\frac{1}{2}\right) \operatorname{arctg} \frac{\eta}{\lambda+1}\right\}\left[1+O\left(\frac{1}{\eta}\right)\right] .
$$


Заметим, что слагаемое этой асимптотики, содержащее арктангенс, обращается в нуль только в случае $\lambda=-1 / 2$, который реализуется лишь при $d=2$.

Теперь из определения (5) множителя $B_{\mu}$ выведем два представления. Для этого символами $\delta_{\mu}(\eta)$ и $\widetilde{\delta}_{\mu}(\eta)$ обозначим аргумент функции $\Gamma(\mu+1+i \eta)$ и сумму, заключенную в равенстве (16) в фигурные скобки в случае $\lambda=\mu$. Затем воспользуемся формулой Муавра и свойствами (15). В итоге при четном $d$ получаем представление

$$
B_{\mu}(\eta)=\frac{\sqrt{2 \pi}}{\Gamma(\mu+1)} \frac{e^{i \delta_{\mu}(\eta)}}{\sqrt{e^{2 \pi \eta}+1}} \prod_{k=1}^{\mu+1 / 2}\left[(\mu+1-k)^{2}+\eta^{2}\right]^{1 / 2}
$$

а при нечетном $d$ - представление

$$
B_{\mu}(\eta)=\frac{\sqrt{2 \pi \eta}}{\Gamma(\mu+1)} \frac{e^{i \delta_{\mu}(\eta)}}{\sqrt{e^{2 \pi \eta}-1}} \prod_{k=1}^{\mu}\left[(\mu+1-k)^{2}+\eta^{2}\right]^{1 / 2} .
$$

Из таких представлений следуют искомые асимптотики

$$
B_{\mu}(\eta) \sim \frac{\sqrt{2 \pi}}{\Gamma(\mu+1)}|\eta|^{\mu+1 / 2} e^{i \widetilde{\delta}_{\mu}(\eta)} \begin{cases}e^{-\pi \eta}, & \eta \rightarrow \infty \\ (-2 \pi \eta)^{p}, & \eta \rightarrow-\infty\end{cases}
$$

в которых по определению $p=0$ при четном $d$ и $p=-1 / 2$ при нечетном $d$.

Асимптотику множителя $C_{\lambda}$ найдем тем же способом, который использовался для вывода асимптотики множителя $B_{\mu}$. Из определения $(9)$ множителя $C_{\lambda}$ в силу свойств (15) следует, что

$$
C_{\lambda}(\eta) \sim \frac{\sqrt{\pi}}{\Gamma(2 \lambda+2)}|2 \eta|^{\lambda+1 / 2} \begin{cases}e^{-\pi \eta}, & \eta \rightarrow \infty \\ (-2 \pi \eta)^{p}, & \eta \rightarrow-\infty\end{cases}
$$

где $p=0$ при четном $d$ и $p=-1 / 2$ при нечетном $d$.

Отметим одну особенность найденных асимптотик множителей $B_{\mu}$ и $C_{\lambda}$ : в случае кулоновского притяжения $(\eta<0)$ эти асимптотики содержат множитель $(-2 \pi \eta)^{p}$, равный единице при четном $d$ и функции $(-2 \pi \eta)^{-1 / 2}$ при нечетном $d$.

\section{5. ЗАКЛЮЧЕНИЕ}

Просуммируем основные результаты настоящей работы, полученные впервые. Для всех волновых функций $\Psi_{\mu}^{\mathrm{c}}$ и $F_{\lambda}$ кулоновского рассеяния квантовой частицы $p_{1}$ в пространстве $\mathbb{R}^{d}$ произвольной размерности $d=2,3, \ldots$ выведены и исследованы разложения (10)-(14) по функциям Бесселя первого рода. Доказано и подтверждено вычислениями следующее утверждение: конечные суммы $\Psi_{\mu m}^{\mathrm{c}}$ и $F_{\lambda m}$ таких разложений являются низкоэнергетическими $(q \rightarrow 0+)$ асимптотиками функций $\Psi_{\mu}^{\mathrm{c}}$ и $F_{\lambda}$ в области $\rho<\infty$.

\section{Список литературы}

[1] D. Yafaev, "On the classical and quantum Coulomb scattering", J. Phys. A: Math. Gen., 30:19 (1997), 6981-6992.

[2] В. В. Пупышев, “Двумерное кулоновское рассеяние квантовой частицы: низкоэнергетические асимптотики", ТМФ, 188:1 (2016), 49-75. 
[3] Г. Бейтмен, А. Эрдейи, Высшие трансиендентные функции, т. 1: Гипергеометрическая функиия. Функиии Лежандра, Наука, М., 1973.

[4] В. В. Пупышев, Кулоновское расеяние медленной квантовой частицы в пространстве произвольной размерности, Препринт Р4-2016-83, ОИЯИ, Дубна, 2016.

[5] Р. И. Джибути, К. В. Шитикова, Метод гиперсферических функций в атомной и ядерной физике, Энергоатомиздат, М., 1993.

[6] J. Avery, Hyperspherical Harmonics. Application in Quantum Theory, Reidel Texts in the Mathematical Sciences, 5, Springer, Dordrecht, 1989.

Поступила в редакцию 25.11.2016, после доработки 31.05.2017 